\title{
Pulmonary Vein Pulsatility in Fetuses of Diabetic Mothers. Prenatal Doppler Echocardiographic Study
}

\author{
Paulo Zielinsky, Antonio L. Piccoli Jr, Lucas Teixeira, Eduardo I. Gus, João L. Mânica, \\ Fabíola Satler, Humberto Vaz, Luiz H. Nicoloso, Stelamaris Luchese, Marlui Sheid, \\ Silvana Marcantonio, Domingos Hatém \\ Porto Alegre, RS - Brazil
}

\begin{abstract}
Objective - To verify the hypothesis that the pulmonary vein pulsatility index is higher in fetuses of diabetic mothers than it is in normal fetuses of nondiabetic mothers.

Methods - Twenty-four fetuses of mothers with either gestational or previous diabetes (cases), and 25 normal fetuses of mothers without systemic disease (control) were examined. Fetuses were examined through prenatal Doppler and color flow mapping. The pulmonary vein pulsatility index was obtained by placing the pulsed Doppler sample volume over the right superior pulmonary vein and applying the formula (systolic velocity - presystolic velocity)/mean velocity.
\end{abstract}

Results - The mean gestational age of the study fetuses was $30.3 \pm 2.7$ weeks, and gestational age of the controls was $29 \pm 3.3$ weeks, with no significant difference in gestational age between groups $(p=0.14)$. Fetuses of diabetic mothers had a mean pulmonary vein pulsatility index of $1.6 \pm 1$, and those of the control group had an index of $0.86 \pm 0.27$.

Conclusion - Fetuses of diabetic mothers had pulmonary vein pulsatility indexes (parameter easily obtained through Doppler echocardiography that may be related to fetal diastolic function) higher than those in fetuses of mothers with normal glycemia.

Key words: fetal echocardiography, fetal diastolic function, fetal pulmonary venous flow, diabetes, pulmonary vein pulsatility index

Instituto de Cardiologia do Rio Grande do Sul - Fundação Universitária de Cardiologia Mailing address: Paulo Zielinsky - Unidade de Cardiologia Fetal do IC/FUC Av. Princesa Isabel, 395 - Cep 90620-001 - Porto Alegre, RS

E-mail: zielinsky@cardiol.br/pesquisa@ cardnet.tche.br

Received for publication: 11/19/02

Accepted for publication $4 / 29 / 03$
The incidence of insulin-dependent diabetes mellitus is about $0.8 \%$, and gestational diabetes is 3-5\%. Both are evidence of the metabolic disturbances of carbohydrates during pregnancy ${ }^{1}$. The incidence of congenital malformation is 3 to 4 times greater in children from diabetic mothers than in the general population ${ }^{2}$. Among those malformations, $50 \%$ are congenital cardiac diseases ${ }^{3}$. Maternal diabetes is a risk factor for congenital heart disease and an indication for fetal echocardiography $y^{4-12}$.

Maternal hyperglycemia and the excess of glucose transferred to the fetus encourage fetal pancreatic islets to increase the production of insulin, leading to hyperinsulinism, which is responsible for fetal complications. Fetal myocardial hypertrophy is the most frequent abnormality found in newborns from diabetic mothers, and it may be found in up to $35 \%$ of these newborns ${ }^{13}$. The interventricular septum is particularly rich in insulin receptors ${ }^{14}$, which would justify increased hypertrophy in this segment, secondary to myocardial cell hyperplasia and hypertrophy due to the increased synthesis of fat and proteins.

Fetal Doppler echocardiography has increased our knowledge about the cardiocirculatory changes in the prenatal period. Recent studies have shown significant changes in the cardiovascular flow of fetuses from diabetic mothers, especially in pregnancies with inadequate glycemic control ${ }^{15}$.

With the introduction of echocardiography, several clinical studies have demonstrated normal patterns of pulmonary venous flow in children and adults through transesophageal and transthoracic echocardiography ${ }^{16,17}$. The use of the pulmonary vein pulsatility index as a parameter for diastolic function evaluation during fetal life has not yet been reported. Thus, we have tested the hypothesis that the pulmonary vein pulsatility index in fetuses from diabetic mothers is greater than that in fetuses from nondiabetic mothers, based on the idea that a less complacent left ventricle would increase presystolic flow impedance in the pulmonary vein, corresponding to the atrial contraction phases. Consequently, it would increase the pulsatility index in this vessel. 


\section{Methods}

Pregnant women with a gestational age between 25 weeks to term were selected and sent to the Unidade de Cardiologia Fetal - Instituto de Cardiologia do RS/Fundação Universitária de Cardiologia (Fetal Cardiology Unit Cardiology Institute of RS/University Foundation of Cardiology) by several obstetric centers in the city.

Twenty-four fetuses with a gestational age between 25 weeks to term whose mothers had previous or gestational diabetes were examined. The control group was formed by 25 normal fetuses between 25 weeks to term, whose mothers did not have diabetes, also examined sequentially. Fetuses were included in the study when the images obtained were of adequate quality and when cardiac abnormalites apart from septal hypertrophy were excluded.

Fetuses with a gestational age less than 25 weeks, with any other congenital malformation, or those whose mother had a systemic disease other than diabetes mellitus were excluded.

The diagnosis of gestational diabetes was made based on the principles proposed by O'Sullivan and Mahan ${ }^{18}$.

The equipment used was an Acuson ASPEN echocardiography system with a convex transducer 4 to $7 \mathrm{MHz}$ or a 2.25 to $4 \mathrm{MHz}$ phased array transducer, with the capacity to perform bidimensional imaging, M-mode, Doppler and color mapping. Fetal echocardiographic examinations were comprehensive, following the segmental sequential approach ${ }^{19}$, starting in the maternal umbilical region and searching as anatomical referentials the dorsal spine, the liver, and the fetal septum primum. Determination of the atrial situs, the position of the heart in the thorax, the type and mode of atrioventricular and ventriculoarterial connections, the aortic arch, and any associated defects was then performed. Flow analysis was performed with pulsed Doppler and color flow mapping, considering that the pulmonary vein flow has systolic, diastolic, and presystolic phases. The pulmonary vein pulsatility index was obtained by placing the pulsed Doppler sample volume over the right superior pulmonary vein (fig. 1), as near as possible to its junction with the left atrium, applying the formula (systolic velocity presystolic velocity)/mean velocity. To obtain adequate flow velocity curves, $0-2 \mathrm{~m} / \mathrm{s}$ scales and 50 to $100 \mathrm{MHZ}$ filters were used. The measures performed in fetal apnea and those corresponding to the average of 3 assessments were considered. All fetal echocardiograms were recorded on magnetic tape.

Data were compared with the 2-tailed Student $t$ test for independent variables. The statistical significance level was established as 0.05 .

\section{Results}

Mean gestational age of the study fetuses was 30.3 \pm 2.7 weeks, and of the controls was $29 \pm 3.3$ weeks. The difference between gestational ages in the 2 groups was not significant, with $\mathrm{p}=0.14$. Mean septal thickening in the study

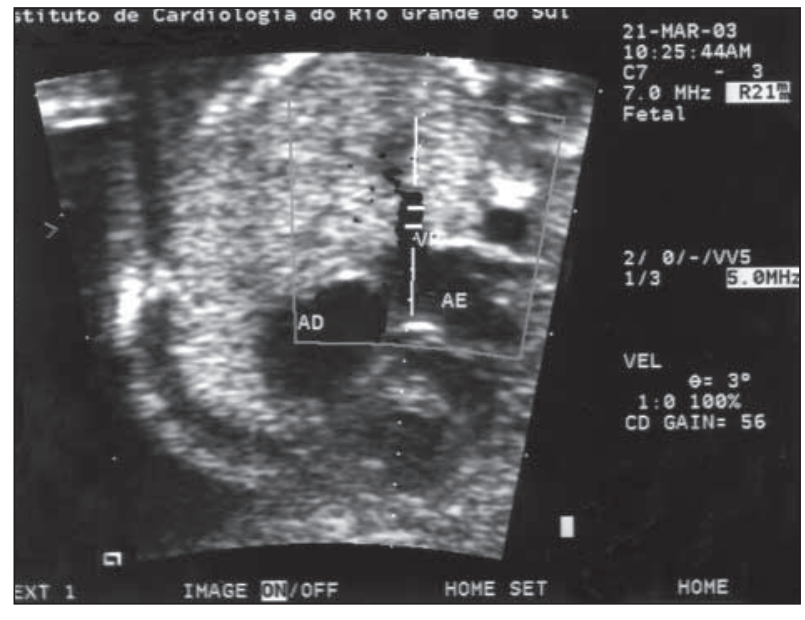

Fig. 1 - Pulmonary vein flow assessment.

fetuses was $3.6 \pm 1 \mathrm{~mm}$, and in the control group it was $2.38 \pm 0.83 \mathrm{~mm}$. In the study fetuses, mean systolic, diastolic, and presystolic velocities were, respectively, $0.29 \pm 0.07 \mathrm{~m} / \mathrm{s}$, $0.21 \pm 0.07 \mathrm{~m} / \mathrm{s}$, and $-0.02 \pm 0.12 \mathrm{~m} / \mathrm{s}$, whereas in the controls they were, respectively, $0.27 \pm 0.05 \mathrm{~m} / \mathrm{s}(\mathrm{NS}), 0.22 \pm 0.05 \mathrm{~m} / \mathrm{s}$ $(\mathrm{NS})$, and $0.10 \pm 0.03 \mathrm{~m} / \mathrm{s}(\mathrm{P}=0.001)$. The mean pulmonary vein pulsatility index in the fetuses of diabetic mothers was $1.6 \pm 1$ (fig. 2) and in the controls, it was $0.86 \pm 0.27$ (fig. 3) with a significant difference ( $\mathrm{p}=0.001$ ) (fig. 4$)$.

\section{Discussion}

The classical approach to the study of ventricular filling function in the fetus is the analysis of atrioventricular flow velocities. We are proposing an alternative method to evaluate fetal diastolic function.

Keren et al ${ }^{20}$ suggested that pulmonary venous flow is influenced by dynamic changes in left atrial pressure created by contraction and relaxation of the atrium and ventricle. The pulmonary vein flow pattern is mainly determined by the events that occur in the left side of the heart ${ }^{20}$. Pul-

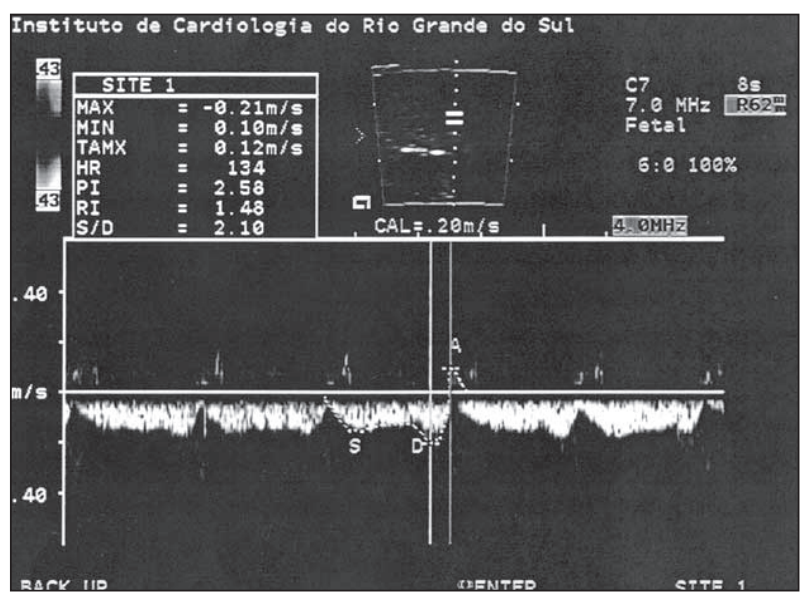

Fig. 2 - Pulmonary vein pulsatility index obtained in fetus of diabetic mother. S, systolic peak; D, diastolic peak; A, presystolic peak 


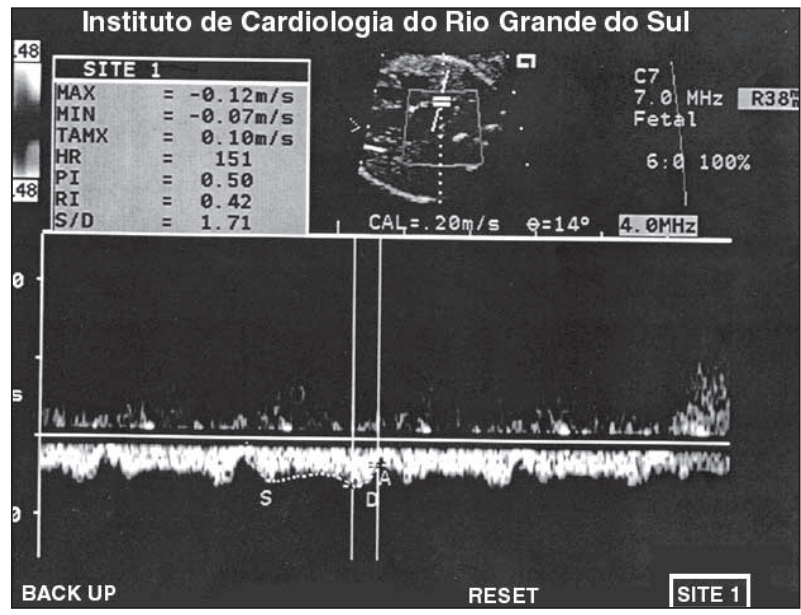

Fig. 3 - Pulmonary vein pulsatility index obtained in fetus of mother with normal glycemia. S, systolic peak; D, diastolic peak; A, presystolic peak.

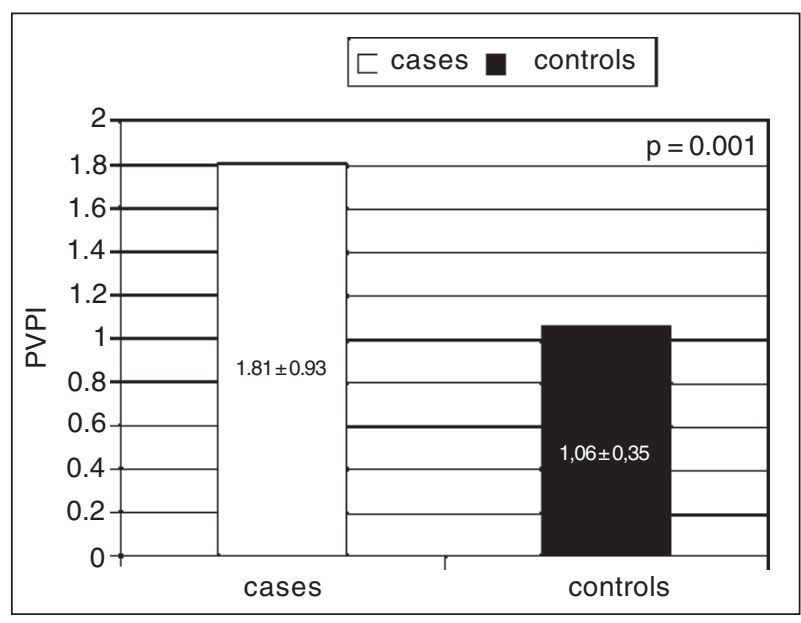

Fig. 4 - Comparison of the pulmonary vein pulsatility index (PVPI) between fetuses of mothers with diabetes and mothers with normal glycemia.

monary venous flow patterns in fetuses and newborns have been studied. Hong and $\mathrm{Choi}^{21}$ reported that pulmonary venous flow was continuous from the pulmonary vein to the left atrium in all cardiac cycles and had biphasic peaks: one during systole and the other during diastole. Fetuses have a pulmonary vein pattern similar to that described in the postnatal period, apart from the absence of reverse atrial flow. As the lungs are collapsed in the fetus, the flow is reduced and, additionally, lower velocities may reflect the decreased pulmonary flow volume. Hong and Choi ${ }^{22}$ verified that the pattern of pulmonary vein flow in fetuses results from the low pulmonary flow and the reduced capacity of the pulmonary system. The pattern of the pulmonary venous waveform was also described by Laudy et al ${ }^{23}$, and reinforces that it has a biphasic flow profile with a systolic and a diastolic component. The nature of the fetal pulmonary venous flow waveform pattern suggests positive pressures towards the left atrium thorough the cardiac cycle.

The pattern of pulmonary venous flow velocity, may be altered in many physiologic and pathologic situations, such as heart rate ${ }^{24}$, cardiac output ${ }^{25}$, absolute pulmonary flow ${ }^{26}$, left atrial pressure ${ }^{22}$ and function, mitral valve function, left ventricle pressure and function ${ }^{22}$. Talbert and Johnson ${ }^{27}$ suggested that the change of shape of the pulmonary venous flow waveform reflects the changes in the pressure waveform of the left atrium and represent the atrial pressure response to both intracardiac and systemic vascular status.

The present study has demonstrated that fetuses from diabetic mothers have a higher pulmonary vein pulsatility index than fetuses from mothers with normal glycemia. It is suggested that this alteration is due to the involvement of fetal diastolic function secondary to previous or gestational maternal diabetes, with left ventricular hypertrophy, and an increase in muscle mass and a consequent decrease in ventricular compliance. The increase in left atrial pressure leads to a restriction of pulmonary venous emptying, resulting in a decrease in presystolic velocity in pulmonary vein or reverse flow in presystole. The marker of this retrograde transmission of pressure would be the increase in pulmonary vein pulsatility index, because this index reflects the correlation between systolic and presystolic velocities, and the mean pulmonary venous flow velocity.

The pulmonary vein pulsatility index is an easily obtained echocardiographic Doppler parameter, which may add information about fetal circulatory dynamics in fetuses from diabetic mothers.

\section{References}

1. Schafer G, Vetter K. Diabetes and pregnancy. Ther Umsch 1999;56: 572-6.

2. Macklon NS, Hop WCJ, Wladimiroff JW. Fetal cardiac function and septal thickness in diabetic pregnancy: a controlled observational and reproducibility study. Br J Obstet Gynaecol 1998;105: 661-6.

3. Goto MP, Goldman AS. Diabetic embryopathy. Curr Opin Pediatr 1994;6:486-91.

4. Behle I, Zielinsky P, Zimmer L, Pontremoli M, Risch J. Níveis de hemoglobina glicosilada e anomalias cardíacas em fetos de mães com diabetes mellitus. RBGO 1998;20: 237-43.

5. Allan LD. Fetal congenital heart disease: diagnosis and management. Curr Opin Obstet Gynecol 1994;6:45-9.

6. Ramos-Arroyo MA, Rodriguez-Pinilla E, Cordero JF. Maternal diabetes: the risk for specific birth defects. Eur J Epidemiol 1992;8:503-8.
7. Albert TJ, Landon MB, Wheller JJ, Samuels P, Cheng RF, Gabbe S. Prenatal detection of fetal anomalies in pregnancies complicated by insulin-dependent diabetes mellitus. Am J Obstet Gynecol 1996;174:1424-8.

8. Becerra JE, Khoury MJ, Cordero JF, Erickson JD. Diabetes mellitus during pregnancy and the risks for specific birth defects: a population-based case-control study. Pediatrics 1990;85:1-9.

9. Gladman G, McCrindle BW, Boutin C, Smallhorn JF. Fetal echocardiographic screening of diabetic pregnancies for congenital heart disease. Am J Perinatol 1997; 14:59-62.

10. Shields LE, Gan EA, Murphy HF, Sahn DJ, Moore TR. The prognostic value of hemoglobin A1c in predicting fetal heart disease in diabetic pregnancies. Obstet Gynecol 1993;81:954-7. 
11. Meyer-Wittkopf M, Simpson JM, Sharland GK. Incidence of congenital heart defects in fetuses of diabetic mothers: a retrospective study of 326 cases. Ultrasound Obstet Gynecol 1996;8:8-10.

12. Smith RS, Comstock CH, Lorenz RP, Kirk JS, Lee W. Maternal Diabetes Mellitus: which views are essential for fetal echocardiography? Obstet Gynecol 1997:90:575-9.

13. Zielinsky P, Costa Mhl, Oliveira LT, Bonow FP, Silva NIT, Hagemann LL. Estudo da história natural da hipertrofia miocárdica e sua associação com hiperinsulinismo em filhos de mães diabéticas. Arq Bras Cardiol 1997;69:389-94.

14. Thorsson AV, Hintz RL. Insulin receptors in the newborn: increase in receptor affinity and number. N Engl J Med 1977;297:908-12.

15. Zielinsky P, Hagemann LL, Daudt LE, Behle I. Pre- and postnatal analysis of factors associated with fetal miocardial hypertrophy in diabetic pregnancies. J Matern Fetal Invest 1992;2:163-7.

16. keren G, Meisner JS, Sherez J, Yellin EL, Laniado S. Iterrelationship of mid-diastolic mitral valve motion, pulmonary vemous flow and transmitral flow. Circulation 1986;74:36-44.

17. Kuecherer HF, Muhiuddem IA, Kusumoto FM, LEEE, Cahalan MK, Shiller NB. Estimation of mean left atrial pressure from trans-esophageal pulsed Doppler echocardiography of pulmonary venous flow. Circulation 1990;82:1127-39.

18. O' Sullivan JB, Mahan CM. Criteria for the oral glucose tolerance test in pregnancy. Diabetes 1964;13:278-85.
19. Zielinsky P, Haertel JC, Lucchese F. Abordagem seqüencial das cardiopatias congênitas: um enfoque ecocardiográfico bidimensional. Arq Bras Cardiol 1985;45:129-44.

20. Keren G, Sherez J, Megidish R, et al. Pulmonary venous flow pattern - its relationship to cardiac dynamics. Circulation 1985;71:1105-12.

21. Hong Y, Choi J. Doppler study on pulmonary venous flow in human fetus. Fetal Diagn Ther 1999;14:86-91

22. Hong YM, Choi JY. Pulmonary venous flow from fetal to neonatal period. Early Hum Dev 2000;57:95-103.

23. Laudy JA, Huisman TW, de Ridder MA, Wladimiroff JW. Normal fetal pulmonary venous blood flow velocity. Ultrasound Obstet Gynecol 1996;7:299-300.

24. Drihashi K, Goliner PL, Oka Y. Intraoperative assessment of pulmonary vein flow. Echocardiography 1990;7:261-71.

25. Kenny JF, Plappert T, Cartier M, et al. Changes in intracardiac blood flow velocities and right and left ventricular stroke volumes with gestational age in the normal human fetus: a prospective Doppler Echocardiography study. Circulation 986;74:1208-16.

26. Wang J, Deng Y, Ying W, Wang X. Pulmonary venous flow evaluated by Doppler Echocardiography. Jpn Heart J 1989;1:55-63.

27. Talbert DG, Johnson P. The pulmonary vein Doppler waveform: feature analysis by comparison of in vivo pressures and flows with those in a computerized fetal physiological model. Ultrasound Obstet Gynecol 2000;16:457-67.

Bula resumida - MICARDIS ${ }^{\circledast}$ Telmisartam - Uso adulto - Forma farmacêutica e apresentações: Comprimidos de $40 \mathrm{mg}$ : embalagens com 14 e 28 comprimidos. Comprimidos de 80 mg: embalagens com 14 e 28 comprimidos. Composição: Cada comprimido contém $40 \mathrm{mg}$ ou $80 \mathrm{mg}$ de telmisartam. Excipientes q.s.p. 1 comprimido. Indicações: Tratamento da hipertensão arterial, como monoterapia ou em associação com outros agentes anti-hipertensivos. Contra-indicações: Hipersensibilidade ao ingrediente ativo ou aos excipientes. Gravidez e lactação. Obstrução biliar. Disfunção hepática ou renal grave. Intolerância hereditária à frutose. Precauções: Hipertensão renovascular: pacientes com estenose arterial renal bilateral ou estenose da artéria com um único rim funcionando: risco aumentado de hipotensão grave e insuficiência renal. Disfunção renal ou transplante hepático: monitoração periódica dos níveis séricos de potássio e creatinina. Não há experiência em pacientes com transplante renal recente. Desidratação: hipotensão
sintomática, especialmente após a primeira dose, pode ocorrer em pacientes que têm volemia e/ou sódio depletado, o que deve ser corrigido antes do início da terapêutica com MICARDIS. Outras condiçóes de sintomática, especialmente após a primeira dose, pode ocorrer em pacientes que têm volemia e/ou sódio depletado, o que deve ser corrigido antes do início da terapêutica com MICARDIS. Outras condições de estimulação do SRAA e condições dependentes da atividade SRAA (insuficiência cardíaca congestiva grave): hipotensão aguda, hiperazotemia, oligúria ou, raramente, insuficiência renal aguda.
Hiperaldosteronismo primário: não se recomenda o uso de MICARDIS. Estenose valvar aórtica e mitral e cardiomiopatia hipertrófica obstrutiva: Recomenda-se precaução especial. Hipercalemia: recomendaHiperaldosteronismo primário: não se recomenda o uso de MICARDIS. Estenose valvar aórtica e mitral e cardiomiopatia hipertrófica obstrutiva: Recomenda-se precaução especial. Hipercalemia: recomendaos níveis de potássio, como a heparina: podem levar a um aumento da potassemia. Portanto, nestas situações MICARDIS deve ser administrado com cautela. Distúrbios hepatobiliares: pode-se esperar redução da depuração em pacientes com disfunçōes obstrutivas do sistema biliar ou insuficiência hepática, pois a eliminação da droga é principalmente biliar. Intolerância à frutose: os comprimidos de MICARDIS contém sorbitol; portanto, é inadequado para pacientes com intolerância hereditária à frutose. Outros: menor eficácia na redução da pressão arterial na população negra do que na população não-negra. Cardiopatia isquêmica ou doença cardiovascular isquêmica pode resultar em infarto do miocárdio. Interaçoes medicamentosas: MICARDIS pode aumentar o efeito hipotensor de outros agentes anti-hipertensivos. Observouse um aumento de $20 \%$ da concentraçao plasmática media de digoxina. Relataram-se aumentos reversiveis das concentraçoes sericas de lítio e de toxicidade; portanto, recomenda-se cuidadosa monitoração urinário, infecçōes do trato respiratório superior, ansiedade, visão anormal, vertigem, dor abdominal, diarréia, boca seca, dispepsia, flatulência, dor de estômago, eczema, aumento de suor, artralgia, dor nas costas, cãibras nas pernas ou dores nas pernas, mialgia, sintomas de tendinite, dor no peito, sintomas de gripe. Além disso, desde a introdução de telmisartam no mercado, relataram-se casos raros de eritema, prurido, desmaio, insônia, depressão, vômito, hipotensão, bradicardia, taquicardia, dispnéia, eosinofilia, trombocitopenia, fraqueza e perda de eficácia. Relataram-se casos isolados de angioedema, urticária e outros eventos relacionados. Investigações: Raramente, observaram-se diminuição na hemoglobina ou aumento no ácido úrico. Observaram-se aumentos na creatinina ou nas enzimas hepáticas. Efeitos na habilidade de dirigir e utilizar máquinas: Ainda não se realizaram estudos específicos. Contudo, ao dirigir ou operar máquinas, pode ocasionalmente ocorrer tontura ou sonolência. Posologia: A dose recomendada é de $40 \mathrm{mg}$ uma vez ao dia. Alguns pacientes podem apresentar benefício com dose diária de $20 \mathrm{mg}$. Em casos em que a pressão arterial pretendida não seja atingida, a dose de MICARDIS pode ser aumentada para no máximo $80 \mathrm{mg}$ uma vez ao dia. Alternativamente, MICARDIS pode ser usado em combinação com diuréticos tiazídicos, como a hidroclorotiazida, para se obter uma redução maior da pressão arterial. Quando se considerar um aumento da dose, deve-se levar em conta que o máximo efeito anti-hipertensivo é geralmente atingido quatro a oito semanas após o início do tratamento. MICARDIS pode ser administrado com ou sem alimento. Insuficiência renal: Não há necessidade de ajustes de dose em pacientes com insuficiência renal leve a moderada. Telmisartam não é removido do sangue por hemofiltração. Insuficiência hepática: Nos pacientes portadores de insuficiência hepática leve a moderada, não se deve exceder a dose diária de 40 mg. Pacientes idosos: Não são necessários ajustes de doses. Crianças e adolescent

Bula resumida - MICARDIS ${ }^{\oplus}$ HCT - Telmisartam/Hidroclorotiazida - Uso adulto - Composição: Cada comprimido de MICARDIS HCT contém $40 \mathrm{mg} / 12,5 \mathrm{mg}$ ou $80 \mathrm{mg} / 12,5 \mathrm{mg}$ de telmisartam/hidroclorotiazida. Embalagens com 14 e 28 comprimidos. Excipientes q.s.p. 1 comprimido. Indicações: Tratamento da hipertensão arterial. MICARDIS HCT, como associação de dose fixa, é indicado em pacientes cuja pressão arterial não é adequadamente controlada com telmisartam ou hidroclorotiazida isoladamente. Posologia: MICARDIS HCT deve ser administrado uma vez ao dia. A dose de MICARDIS pode ser aumentada gradativamente antes de substituí-lo pelo MICARDIS HCT. A substituição direta da monoterapia pelas combinações fixas pode ser considerada. MICARDIS HCT 40/12,5 mg pode ser administrado em pacientes cujas pressões sangüíneas não sejam adequadamente controladas por MICARDIS $40 \mathrm{mg}$ ou hidroclorotiazida. MICARDIS HCT 80/12,5 mg pode ser administrado em pacientes cujas pressões arteriais não sejam adequadamente controladas por MICARDIS $80 \mathrm{mg}$ ou por MICARDIS HCT 40/12,5 mg. O máximo efeito anti-hipertensivo é obtido após 4 a 8 semanas de tratamento. Quando necessário, MICARDIS HCT pode ser administrado com outros anti-hipertensivos. MICARDIS HCT pode ser administrado com ou sem alimento. Devido ao componente hidroclorotiazida, MICARDIS HCT não deve ser usado em pacientes com
disfunção renal grave. Nos casos leves a moderados, não se observaram efeitos adversos renais e não é necessário ajuste de dose. Recomenda-se monitoração periódica da função renal. Nos casos de
insuficieiencia hepática leve a moderada, não se deve exceder a dose de $40 / 12,5 \mathrm{mg}$ uma vez ao dia. MICARDIS HCT não é indicado em pacientes com insuficiência hepática grave. Não são necessários ajustes insuficiência hepática leve a moderada, não se deve exceder a dose de 40/12,5 mg uma vez ao dia. MICARDIS HCT não é indicado em pacientes com insuficiência hepática grave. Não são necessários ajustes de dose conforme a idade. Ainda não se estabeleceram a segurança e a eficácia de MICARDIS HCT em pacientes menores de 18 anos. Contra-indicações: Hipersensibilidade aos componentes ativos,
excipientes ou substâncias derivadas de sulfonamidas (como HCT). Gravidez e lactação. Colestase e distúrbios obstrutivos biliares. Insuficiência hepática ou renal grave (depuração de creatinina <30 ml/min). excipientes ou substâncias derivadas de sulfonamidas (como HCT). Gravidez e lactação. Colestase e distúrbios obstrutivos biliares. Insuficiência hepática ou renal grave (depuração de creatinina <30 ml/min). Hipopotassemia refratáría e hipercalcemia. Precauções: Năo utilizar nos casos de colestase, distúrbios biliares obstrutivos ou insuficiência hepática grave. Usar com cautela nos casos de função hepática
alterada ou doença hepática progressiva, pois pode ocorrer coma hepático. Há maior risco em pacientes com estenose arterial renal bilateral ou estenose com um único rim funcionando. Micardis HCT não deve ser administrado em pacientes portadores de disfunção renal grave. Não há experiência quanto à administração de MICARDIS HCT em pacientes com grave insuficiência renal ou com um rim transplantado ser administrado em pacientes portadores de disfunção renal grave. Não há experiência quanto à administração de MICARDIS HCT em pacientes com grave insuficiência renal ou com um rim transplantado Pode ocorrer hipotensão sintomática em pacientes que têm volemia e/ou sódio depletado. Insuficiência cardíaca congestiva grave ou doença renal, estenose da artéria renal tratada concomitantemente com drogas que afetem o sistema renina-angiotensina-aldosterona foram associados com hipotensão aguda, hiperazotemia, oligúria ou, raramente, insuficiência renal aguda. Aldosteronismo primário: não se ajuste no tratamento em diabéticos e pode ocorrer manifestação de diabetes latente. A hidroclorotiazida aumenta os níveis de colesterol e triglicérides; contudo, nenhum ou poucos efeitos adversos foram relatados na dose de $12,5 \mathrm{mg}$ contida em MICARDIS HCT. Pode ocorrer hiperuricemia ou precipitação de gota devido a hidroclorotiazida. Pode ser necessária monitoração periódica dos níveis séricos de eletrólitos, uma vez que a hidroclorotiazida pode causar desequilíbrio eletrolítico ou de fluidos (hipopotassemia, hiponatremia e alcalose hipoclorêmica). O tratamento concomitante com telmisartam pode reduzir a hipopotassemia induzida por diuréticos, devido aos efeitos antagônicos. Diuréticos poupadores de potássio, suplementos de potássio ou substitutos do sal contendo potássio devem ser utilizados concomitantemente com cautela. Nẫo há evidências de que MICARDIS HCT reduza ou previna hiponatremia induzida por diuréticos. A deficiência de cloreto é geralmente leve. Tiazidas podem causar elevação dos níveis séricos de cálcio e magnésio. Interromper o uso antes dos testes de função da paratireóide. Não utilizar em intolerância hereditária à frutose. A redução excessiva da pressão arterial no caso de doença cardiovascular isquêmica pode ser prejudicial. Relatou-se exacerbação ou ativação do lupus eritematoso sistêmico com o uso de hidroclorotiazida. Recomenda-se cautela no uso de anti-hipertensivos ao dirigir e operar máquinas. Interações medicamentosas: Relataram-se aumentos reversíveis das concentrações séricas de lítio durante administração concomitante com inibidores da enzima conversora de
angiotensina. Relataram-se casos de interação com antagonistas dos receptores da angiotensina II. O risco de toxicidade por lítio pode ser aumentado com o uso de MICARDIS HCT. Recomenda-se a monitoração
dos níveis séricos de lítio durante o uso concomitante. O efeito de depleção de potássio da hidroclorotiazida é atenuado pelo efeito poupador de potássio do telmisartam, mas é potencializado por outros diuréticos dos níveis séricos de lítio durante o uso concomitante. O efeito de depleção de potássio da hidroclorotiazida é atenuado pelo efeito poupador de potássio do telmisartam, mas é potencializado por outros diuréticos caliuréticos, laxantes, corticosteróides, ACTH, anfotericina, carbenoxolona, penicilina G sódica, ácido salicílico e derivados. Por outro lado, o uso concomitante de diuréticos poupadores de potássio,
suplementos/sais de potássio ou outras drogas podem aumentar os níveis séricos de potássio. Nesses casos, recomenda-se a monitoração dos níveis plasmáticos de potássio. Recomenda-se monitoração
periódica de potássio na administração de MICARDIS HCT com digitálicos, antiarrítmicos e drogas que são conhecidamente indutoras de torsades de pointes. Telmisartam pode aumentar o efeito hipotensor de outros agentes anti-hipertensivos. Observou-se um aumento de $20 \%$ da concentração plasmática média de digoxina. Não se identificaram outras interações de importância clínica. Interaçōes com a hidroclorotiazida: álcool, barbitúricos ou narcóticos (potencialização da hipotensão ortostática); drogas antidiabéticas (ajustes de dose podem ser necessários); mettormina (risco de acidose láctica); (hipopotassemia ou hipomagnesemia induzida por tiazídicos favorece o aparecimento de arritmias cardíacas induzidas por digitálicos); drogas antiinflamatórias não-esteroidais (a administração de droga diminuídos. Os relaxantes musculares esqueléticos não-despolarizantes são potencializados pela hidroclorotiazida. Medicamentos uricosúricos podem requerer ajustes. Verificou-se aumento da incidência de reações de hipersensibilidade ao alopurinol. Os efeitos hiperglicêmicos dos beta-bloqueadores e diazóxido podem ser aumentados pelas tiazidas. Os agentes anticolinérgicos (por exemplo, atropina, biperideno) podem aumentar a biodisponibilidade das tiazidas. As tiazidas podem aumentar os eventos adversos da amantadina. As tiazidas podem reduzir a excreção renal de drogas citotóxicas (por exemplo, ciclofosfamida, metotrexato). Gravidez e lactação: É contra-indicado. Se ocorrer gravidez, telmisartam deve ser descontinuado o mais breve possível. As tiazidas atravessam a barreira placentária e podem causar distúrbios eletrolíticos no feto e é possível que ocorram outras reações. Relataram-se casos de trombocitopenia ou icterícia neonatal com o tratamento tiazídico materno. Tiazidas são excretadas no leite humano e podem inibir a lactação. Reações adversas: Foram relatadas as seguintes reações adversas: Bronquite, faringite, sinusite, infecções do trato respiratório superior, infecções do trato urinário, sialadenite, eosinofilia, anemia aplástica, anemia hemolítica, depressão da medula óssea, leucopenia, neutropenia/agranulocitose, trombocitopenia, alergia, reações anafilíticas, perda do controle da diabete, hipercolesterolemia, hiperuricemia, hipopotassemia, causa ou aumento da depleção de volume, desequilíbrio eletrolítico, hiponatremia, anorexia, perda de apetite, hiperglicemia, ansiedade, depressão,
inquietação, tontura, desmaio, insônia, escotomas, parestesia, distúrbios do sono, visão alterada, visão borrada temporariamente, xantopsia, vertigem, bradicardia, taquicardia, arritmias cardíacas, hipotensão, inquietação, tontura, desmaio, insônia, escotomas, parestesia, distúrbios do sono, visão alterada, visão borrada temporariamente, xantopsia, vertigemem, bradicardia, taquicardia, arritmias cardíacas, hipotensão,
hipotensão postural, angiite necrotizante (vasculite), dispnéia, sofrimento respiratório (incluindo pneumonite e edema pulmonar), dor abdominal, diarréia, dispepsia, gastrite, dor de estômago, boca seca, hipotensão postural, angiite necrotizante (vasculite), dispnéia, sofrimento respiratório (incluindo pneumonite e edema pulmonar), dor abdominal, diarréia, dispepsia, gastrite, dor de estômago, boca seca,
flatulência, vômito, constipação, pancreatite, icterícia (icterícia hepatocelular ou colestática), eczema açónes flatulência, vômito, constipação, pancreatite, icterícia (icterícia hepatocelular ou colestática), eczema, aumento do suor, eritema, prurido, reações cutâneas do tipo lupus eritematoso, vasculite cutânea, reações
de fotossensibilidade, erupcões cutâneas, reativação do lupus eritematoso cutâneo, necrólise epidérmica tóxica, atralgia, artrose, dor nas costas, dor nas pernas, mialgia, cãibras nas pernas, sintomas de de fotossensibilidade, erupções cutâneas, reativação do lupus eritematoso cutâneo, necrólise epidérmica tóxica, atralgia, artrose, dor nas costas, dor nas pernas, mialgia, cãibras nas pernas, sintomas de
tendinite, fraqueza, espasmo muscular, nefrite intersticial, disfunção renal, glicosúria, impotência, sintomas de gripe, dor, dor no peito, perda da eficácia, febre. Investigações: Diminuição da hemoglobina, aumento tendinite, fraqueza, espasmo muscular, nefrite intersticial, disfunção renal, glicosúria, impotência, sintomas de gripe, dor, dor no peito, perda da eficácia, febre. Investigações: Diminuição da hemoglobina, aumento
do ácido úrico, aumento na creatinina, aumento das enzimas hepáticas, aumento dos triglicérides. Assim como ocorre com outros antagonistas da angiotensina II, relataram-se casos isolados de angioedema, do ácido úrico, aumento na creatinina, aumento das enzimas hepáticas, aumento dos triglicérides. Assim como ocorre com outros antagonistas da angiotensina
urticária e outras reações relacionadas. VENDA SOB PRESCRIÇÂO MÉDICA. - MS 1.0367.0134 Boehringer Ingelheim do Brasil Química e Farmacêutica Ltda. 